TITLE:

\title{
Gray-box modeling for prediction and control of molten steel temperature in tundish
}

$\operatorname{AUTHOR}(S)$ :

Ahmad, Iftikhar; Kano, Manabu; Hasebe, Shinji; Kitada, Hiroshi; Murata, Noboru

\section{CITATION:}

Ahmad, Iftikhar ...[et al]. Gray-box modeling for prediction and control of molten steel temperature in tundish. Journal of Process Control 2014, 24(4): 375-382

\section{ISSUE DATE:}

2014-04

URL:

http://hdl.handle.net/2433/187099

\section{RIGHT:}

(C) 2014 Elsevier Ltd.; This is not the published version. Please cite only the published version.; この論文は出版社版でありません。引用の際に は出版社版をご確認ご利用ください。 


\title{
Gray-Box Modeling for Prediction and Control of Molten Steel Temperature in Tundish
}

\author{
Iftikhar Ahmad $^{\mathrm{a}}$, Manabu Kano*b ${ }^{*}$, Shinji Hasebe ${ }^{\mathrm{a}}$, Hiroshi Kitada ${ }^{\mathrm{c}}$, Noboru \\ Murata $^{\mathrm{d}}$ \\ ${ }^{a}$ Dept. of Chemical Engineering, Kyoto University, Nishikyo-ku, Kyoto, 615-8510, Japan \\ ${ }^{b}$ Dept. of Systems Science, Kyoto University, Yoshida-Honmachi, Sakyo-ku, Kyoto, \\ 606-8501, Japan \\ ${ }^{c}$ Nippon Steel and Sumitomo Metal Corporation, Ltd., 1-8 Fusocho, Amagasaki-shi, \\ Hyogo, 660-0891, Japan \\ ${ }^{d}$ Dept. of Electrical Engineering and Bioscience, Waseda University, 3-4-1 Okubo, \\ Shinjuku-ku, Tokyo, 169-8555, Japan
}

\begin{abstract}
To realize stable production in the steel industry, it is important to control molten steel temperature in a continuous casting process. The present work aims to provide a general framework of gray-box modeling and to develop a gray-box model that predicts and controls molten steel temperature in a tundish (TD temp) with high accuracy. Since the adopted firstprinciple model (physical model) cannot accurately describe uncertainties such as degradation of ladles, their overall heat transfer coefficient, which is a parameter in the first-principle model, is optimized for each past batch separately, then the parameter is modeled as a function of process variables through a statistical modeling method, random forests. Such a model is termed as a serial gray-box model. Prediction errors of the first-principle model or the serial gray-box model can be compensated by using another statistical model; this approach derives a parallel gray-box model or a combined gray-box model. In addition, the developed gray-box models are used to determine the optimal molten steel temperature in the Ruhrstahl-Heraeus degassing process from the target TD temp, since the continuous casting process has no manipulated variable to directly control TD temp. The proposed
\end{abstract}


modeling and control strategy is validated through its application to real operation data at a steel work. The results show that the combined gray-box model achieves the best performance in prediction and control of TD temp and satisfies the requirement for its industrial application.

Keywords: Gray-Box Modeling, Model-Based Control, Steel Making Process, Soft-Sensor, Virtual Sensing

* Corresponding author. Tel.: +81-75-7533367; fax: +81-75-7533371.

E-mail address: manabu@human.sys.i.kyoto-u.ac.jp (M. Kano).

\section{Introduction}

The steel industry faces stiff competition in the global market, and each steel company has to realize stable and efficient operation and produce high quality products satisfying various customer demand [1]. The process diagram of the steel making process is shown in Fig. 1. The tundish is a vessel used for delivering molten steel from a ladle to a mold in the continuous casting process. In steel making, control of the molten steel temperature in the tundish (TD temp) is one of the key factors to realizing stable operation. If TD temp is too high, breakouts may occur and cause tremendous increase in maintenance cost and productivity loss. When the temperature is too low, clogging in the tundish nozzle occurs, which causes disruptions in the casting process. However, no effective manipulated variable is available after the secondary refining process to control TD temp. To realize the target TD temp, therefore, it is necessary to adjust the molten steel temperature in the secondary refining process (Ruhrstahl-Heraeus degassing process). The molten steel temperature at the end of secondary refining operation is hereafter called RH temp. To control TD temp by manipulating RH temp, a model relating TD temp and RH temp needs to be constructed. In the past, various models such as first-principle models [2] $]^{-}[7]$, statistical models [8], and gray-box models $[9]^{-}[12]$ have been proposed. 
The gray-box model, which integrates a first-principle model and a statistical model, has attracted researchers' attention by its capability: known linear/nonlinear phenomena can be embedded in the first-principle model, and an unknown relationship among variables can be embedded in the statistical model by extracting such a relationship from the data. In general, gray-box models are more accurate than simplified first-principle models, less complicated than computational fluid dynamics (CFD) models, and more easily interpreted than statistical models. Although a gray-box model aims to improve the prediction performance by combining a first-principle model and a statistical model, the accuracy of the first-principle model is still important. In general, first-principle models have various parameters which need to be determined by using data. Even when some parameters depend on the operating conditions, they are kept constant if it is difficult to identify the relationship between the parameters and the operating conditions. In such a case, large prediction error might be caused.

The present work aims to develop a new gray-box model that can overcome such deficiency and can predict molten steel temperature with high accuracy. To achieve this goal, a parameter in the first-principle model is estimated from process variables with a nonlinear statistical model. In addition, process disturbances such as uncertainties in temperature measurements, composition and weight of added alloys and the extent of oxidation reactions for removal of impurities are also taken into account. Ideally, such disturbances should be modeled by adding certain mathematical expressions to the first-principle model. However, due to lack of process information, realizing such mathematical expressions is difficult and therefore another statistical model is developed to compensate prediction errors caused by such process disturbances. Random forests (RF) is adopted in this work to build statistical models.

In section 2, three types of gray-box models are explained in general. Then, the first-principle model of the steel making process is described in 
sections 3, and the statistical models integrated with the first-principle model to build the gray-box models are described in section 4 . In section 5 , the proposed method is applied to the problems of predicting and controlling molten steel temperature in a real steel making process. Finally, the contents are summarized in the conclusion section.

\section{Gray-box Models}

A general framework of the gray-box modeling is shown in Fig. 2, where gray-box models are categorized into three types, i.e., parallel gray-box models [9], serial gray-box models [10], and combined gray-box models. In this section, modeling methods of these gray-box models are explained.

\subsection{Parallel Gray-box Model}

A typical gray-box model is constructed by combining a first-principle model and a statistical model sequentially; the statistical model is built so as to compensate the error of the first-principle model. This type of gray-box model, hereafter called the parallel gray-box model, is developed through the following steps.

i. Build a first-principle model $f_{\mathrm{fp}}$ to predict an output variable $y$ from input variables $\boldsymbol{x}_{\mathrm{fp}}$.

$\hat{y}_{\mathrm{fp}}=f_{\mathrm{fp}}\left(\boldsymbol{x}_{\mathrm{fp}}, \boldsymbol{\theta}\right)$

where $\hat{y}_{\mathrm{fp}}$ is the prediction of $y$ and $\boldsymbol{\theta}$ is a parameter vector. The firstprinciple model can be of any form including differential algebraic equations. Eq. (1) can be derived from such a first-principle model as shown in the next section.

ii. Estimate $\boldsymbol{\theta}$ by minimizing the sum of squared errors. 


$$
\begin{aligned}
& \tilde{\boldsymbol{\theta}}=\underset{\boldsymbol{\theta}}{\operatorname{argmin}} \sum_{n=1}^{N_{\mathrm{d}}} e_{\mathrm{fp}, n}^{2} \\
& e_{\mathrm{fp}, n}=y_{n}-f_{\mathrm{fp}}\left(\boldsymbol{x}_{\mathrm{fp}, n}, \boldsymbol{\theta}\right) \\
& \boldsymbol{\theta}_{\mathrm{L}} \leq \boldsymbol{\theta} \leq \boldsymbol{\theta}_{\mathrm{U}}
\end{aligned}
$$

where $\boldsymbol{x}_{\mathrm{fp}, n}$ and $y_{n}$ are the $n$th sample of input and output variables, respectively. $N_{\mathrm{d}}$ is the number of samples used for developing the model. $\boldsymbol{\theta}_{\mathrm{L}}$ and $\boldsymbol{\theta}_{\mathrm{U}}$ are lower and upper bound vectors of parameters which are determined in advance.

iii. Build a statistical model $f_{\mathrm{pa}}$ to predict the output error $e_{\mathrm{fp}}$ from input variables $\boldsymbol{x}$.

$$
\begin{aligned}
& \tilde{\boldsymbol{\varphi}}_{\mathrm{pa}}=\underset{\boldsymbol{\varphi}_{\mathrm{pa}}}{\operatorname{argmin}} \sum_{n=1}^{N_{\mathrm{d}}}\left(e_{\mathrm{fp}, n}-f_{\mathrm{pa}}\left(\boldsymbol{x}_{n}, \boldsymbol{\varphi}_{\mathrm{pa}}\right)\right)^{2} \\
& \hat{e}_{\mathrm{fp}, n}=f_{\mathrm{pa}}\left(\boldsymbol{x}_{n}, \boldsymbol{\varphi}_{\mathrm{pa}}\right)
\end{aligned}
$$

where $\varphi_{\mathrm{pa}}$ is a vector of parameters in the outer statistical model. In general, $\boldsymbol{x}_{\mathrm{fp}}$ is a subset of $\boldsymbol{x}$.

iv. Build a gray-box model by combining the first-principle model and the outer statistical model.

$$
\hat{y}_{\mathrm{pa}}=f_{\mathrm{fp}}\left(\boldsymbol{x}_{\mathrm{fp}}, \tilde{\boldsymbol{\theta}}\right)+f_{\mathrm{pa}}\left(\boldsymbol{x}, \tilde{\boldsymbol{\varphi}}_{\mathrm{pa}}\right)
$$

where $\hat{y}_{\mathrm{pa}}$ is the prediction of $y$ by using the parallel gray-box model.

The parallel gray-box model is the simple sum of the first-principle model and the statistical model. This statistical model is referred to as the outer statistical model because it is used on the outside of the first-principle model. 
In general, the parallel gray-box model can significantly improve the prediction performance because it can extract information from data that is not used in the first-principle model and also it can overcome the limitations imposed by the structure of the first-principle model.

\subsection{Serial Gray-box Model}

In the parallel gray-box model, the optimal parameters $\tilde{\boldsymbol{\theta}}$ are constant, although some parameters depend on the operating conditions. This simplification may deteriorate the prediction performance of the first-principle model. Thus, another type of gray-box model, called the serial gray-box model, is used to estimate the parameters as functions of input variables. The serial gray-box model is constructed by the following steps.

i. Develop a first-principle model $\hat{y}_{\mathrm{fp}}=f_{\mathrm{fp}}\left(\boldsymbol{x}_{\mathrm{fp}}, \boldsymbol{\theta}\right)$.

ii. Estimate the parameters $\boldsymbol{\theta}$ in the first-principle model through Eqs. (2)(4).

iii. Select a parameter $\theta_{i}$ and optimize it for each modeling sample.

$$
\begin{aligned}
& \tilde{\theta}_{i, n}=\underset{\theta_{i, n}}{\operatorname{argmin}}\left(y_{n}-f_{\mathrm{fp}}\left(\boldsymbol{x}_{\mathrm{fp}, n}, \tilde{\boldsymbol{\theta}}_{i}^{c}, \theta_{i, n}\right)\right)^{2} \\
& \quad\left(n=1,2, \cdots, N_{\mathrm{d}} ; i=1,2, \cdots, N_{\mathrm{p}}\right) \\
& \theta_{\mathrm{L}, i} \leq \theta_{i, n} \leq \theta_{\mathrm{U}, i}
\end{aligned}
$$

where $\tilde{\theta}_{i, n}$ is the optimal value of the parameter $\theta_{i}$ for sample $n$ and $\tilde{\boldsymbol{\theta}}_{i}^{c}$ is the constant vector consisting of the estimated parameters except $\theta_{i}$. $N_{\mathrm{p}}$ is the number of parameters in the first-principle model. $\theta_{\mathrm{L}, i}$ and $\theta_{\mathrm{U}, i}$ are lower and upper bounds of the $i$ th parameter which are determined in advance.

iv. Repeat step iii for all the parameters one by one, and select the parameter which achieves the smallest sum of squared errors. 
v. Build a statistical model $f_{\text {se }}$ to estimate the selected parameter $\theta_{i}$ from the input variables $\boldsymbol{x}$.

$$
\tilde{\boldsymbol{\varphi}}_{\mathrm{se}, i}=\underset{\boldsymbol{\varphi}_{\mathrm{se}, i}}{\operatorname{argmin}} \sum_{n=1}^{N_{\mathrm{d}}}\left(\tilde{\theta}_{i, n}-f_{\mathrm{se}}\left(\boldsymbol{x}_{n}, \boldsymbol{\varphi}_{\mathrm{se}, i}\right)\right)^{2}
$$

where $\varphi_{\mathrm{se}, i}$ is a vector of parameters of the inner statistical model.

vi. Build a gray-box model by combining the first-principle model and the inner statistical model.

$$
\begin{aligned}
& \hat{y}_{\mathrm{se}}=f_{\mathrm{fp}}\left(\boldsymbol{x}_{\mathrm{fp}}, \tilde{\boldsymbol{\theta}}_{i}^{c}, \hat{\tilde{\theta}}_{i}\right) \\
& \hat{\tilde{\theta}}_{i}=f_{\mathrm{se}}\left(\boldsymbol{x}, \tilde{\boldsymbol{\varphi}}_{\mathrm{se}, i}\right)
\end{aligned}
$$

The serial gray-box model uses the inner statistical model to update a part of parameters of the first-principle model. The structure of the first-principle model still imposes limitations on the achievable prediction performance, but the serial gray-box model has a potential for the improvement through the parameter update based on available data. This type of gray-box model is useful to enhance the understanding of the process because the model is based on first principles and important physical parameters are identified and related with process variables.

\subsection{Combined Gray-box Model}

By combining the above-mentioned two approaches, i.e., the parallel graybox modeling and the serial gray-box modeling, a combined gray-box model can be developed. In the combined gray-box model, a prediction error of a serial gray-box model is compensated by an outer statistical model. In other words, the combined gray-box model consists of the first-principle model, the inner statistical model to estimate parameters, and the outer statistical model to compensate the prediction error. The combined gray-box model is constructed by the following steps. 
i. Build a serial gray-box model Eqs. (11) and (12) to predict an output variable $y$.

$e_{\mathrm{se}}=y-\hat{y}_{\mathrm{se}}$

where $\hat{y}_{\mathrm{se}}$ is the prediction of $y$ and $e_{\mathrm{se}}$ is the output error of the serial gray-box model.

ii. Build a statistical model $f_{\text {com }}$ to predict the output error $e_{\text {se }}$ from input variables $\boldsymbol{x}$.

$$
\tilde{\boldsymbol{\varphi}}_{\text {com }}=\underset{\boldsymbol{\varphi}_{\text {com }}}{\operatorname{argmin}} \sum_{n=1}^{N_{\mathrm{d}}}\left(e_{\mathrm{se}, n}-f_{\text {com }}\left(\boldsymbol{x}_{n}, \boldsymbol{\varphi}_{\text {com }}\right)\right)^{2}
$$

where $\boldsymbol{\varphi}_{\text {com }}$ is a vector of parameters of the outer statistical model.

iii. Build a combined gray-box model by combining the serial gray-box model and the outer statistical model.

$$
\hat{y}_{\mathrm{com}}=f_{\mathrm{fp}}\left(\boldsymbol{x}_{\mathrm{fp}}, \tilde{\boldsymbol{\theta}}^{c}, \hat{\tilde{\theta}}\right)+f_{\mathrm{com}}\left(\boldsymbol{x}, \tilde{\boldsymbol{\varphi}}_{\mathrm{com}}\right)
$$

where $\hat{y}_{\text {com }}$ is the prediction of $y$ by using the combined gray-box model.

\section{First-Principle Model}

In this research, the molten steel temperature at the end of secondary refining operation, RH temp, is used to control TD temp. After the secondary refining is finished, the molten steel is transfered to the continuous casting process with a ladle. The molten steel is then discharged from the ladle to the tundish so as to execute the continuous casting. The first-principle model which predicts TD temp consists of two sub-models. The first sub-model describes the phenomena during the transportation period, in which the molten 
steel is transfered from secondary refining to continuous casting. The second sub-model describes the phenomena during the discharging period, in which the molten steel is discharged from the ladle to the tundish. The proposed first-principle model is a modified version of the model developed by Okura et al. [12].

\subsection{First-Principle Model for Transportation Period}

\subsubsection{Molten Steel in Ladle}

It is assumed that the ladle is a cylinder of radius $R_{1}$. On the basis of the CFD simulation results, indicating that thermal stratification is formed vertically in the standing ladle due to natural convection [4], the molten steel temperature is modeled as a function of time $t$ and position $z$ from the bottom of the ladle.

$T_{\mathrm{m}}(z, t)=\bar{T}_{\mathrm{m}}(t)+k(t)\left(\sqrt{\frac{z}{H_{\mathrm{m}}}}-\frac{2}{3}\right)$

where $T_{\mathrm{m}}$ is the molten steel temperature, $\bar{T}_{\mathrm{m}}$ is its average, $k$ denotes the difference between the molten steel temperature at the top and the bottom of the ladle, and $H_{\mathrm{m}}$ is the depth of the molten steel in the ladle.

The results of CFD simulations have shown that the temperature difference is a function of time [4]; thus it is modeled with parameter $\alpha$.

$k(t)=\alpha t$

The initial molten steel temperature is assumed to be homogeneous and the same as RH temp because the molten steel in the ladle is properly stirred. Thus, $k(t)=0$ at $t=0$. The transition of average molten steel temperature $\bar{T}_{\mathrm{m}}(t)$ is calculated through the heat balance equation. 


$$
\begin{array}{r}
\rho_{\mathrm{m}} c_{\mathrm{m}} \pi R_{\mathrm{l}}^{2} H_{\mathrm{m}} \frac{d \bar{T}_{\mathrm{m}}(t)}{d t}=-2 \pi R_{\mathrm{l}} \int_{0}^{H_{\mathrm{m}}} U_{\mathrm{w}}\left(T_{\mathrm{m}}(z, t)-T_{\mathrm{am}}\right) d z \\
-\pi R_{\mathrm{l}}^{2} U_{\mathrm{b}}\left(T_{\mathrm{m}}(0, t)-T_{\mathrm{am}}\right)-\pi R_{\mathrm{l}}^{2} h_{1}\left(T_{\mathrm{m}}\left(H_{\mathrm{m}}, t\right)-T_{\mathrm{sl}}(t)\right)
\end{array}
$$

where $\rho_{\mathrm{m}}$ and $c_{\mathrm{m}}$ are the density and the heat capacity of the molten steel, respectively. $U_{\mathrm{b}}$ and $U_{\mathrm{w}}$ are the overall heat transfer coefficients of the ladle bottom and the ladle wall, respectively. $T_{\mathrm{am}}$ and $T_{\mathrm{sl}}$ are the ambient temperature and the slag temperature, respectively. In addition, $h_{1}$ denotes the heat transfer coefficient between the molten steel and the slag. The left side of Eq. (18) represents the time change of the molten steel enthalpy. The first, second, and third terms of the right side represent the heat conduction from the molten steel to the ladle wall, to the ladle bottom, and to the slag, respectively.

The average molten steel temperature is gradually decreased with time by Eq. (18). The temperature profile in the ladle at two different moments is shown in Fig. 3.

\subsubsection{Slag in Ladle}

Slag in the standing ladle, which is generated in the converter, keeps the molten steel at high temperature. The heat balance of the slag is modeled as a lumped parameter system.

$$
\begin{aligned}
\rho_{\mathrm{sl}} c_{\mathrm{sl}} \pi R_{1}^{2} H_{\mathrm{sl}} \frac{d T_{\mathrm{sl}}(t)}{d t}=\pi & R_{\mathrm{l}}^{2} h_{1}\left(T_{\mathrm{m}}\left(H_{\mathrm{m}}, t\right)-T_{\mathrm{sl}}(t)\right)-\pi R_{1}^{2} \varepsilon_{\mathrm{sl}} \sigma\left(T_{\mathrm{sl}}(t)^{4}-T_{\mathrm{a} 1}^{4}\right) \\
& -\pi R_{1}^{2} h_{2}\left(T_{\mathrm{sl}}(t)-T_{\mathrm{a} 1}\right)-2 \pi R_{1} H_{\mathrm{sl}} U_{\mathrm{w}}\left(T_{\mathrm{sl}}(t)-T_{\mathrm{am}}\right)
\end{aligned}
$$

where $\rho_{\mathrm{sl}}, c_{\mathrm{sl}}$, and $\varepsilon_{\mathrm{sl}}$ are the density, the heat capacity, and the emissivity of the slag, respectively. $H_{\mathrm{sl}}$ denotes the slag layer thickness, $h_{2}$ the heat transfer coefficient between the slag and the air in the ladle, $T_{\mathrm{a} 1}$ the air temperature in the ladle, and $\sigma$ the Stefan-Boltzmann coefficient. The left 
side of Eq. (19) represents the time change of the slag enthalpy. The first, second, third and fourth terms of the right side represent the heat conduction from the molten steel to the slag, the radiation from the slag to the wall of the ladle, the heat conduction from the slag to the air in the ladle, and that from the slag to the ladle wall, respectively. The ladle wall temperature, which affects the radiation, is assumed to be equal to the air temperature.

\subsubsection{Ladle Degradation}

On the basis of the knowledge of process engineers and plant operators, the heat loss from the ladle is a key factor to model the process. However, there are two factors that make the modeling difficult. The first one is that the ladle wall gradually degrades due to the repeated use. The second one is that there are multiple ladles with different characteristics. The effect of ladle degradation on the heat conduction flux from the molten steel to the external environment has been discussed in the literature. One study [13] describes the factors which cause degradation of ladle while another study [14] develops a CFD model to relate the heat losses from ladle with the reduction in ladle walls and bottom thickness. To avoid computational complexity and build a simple model, it is assumed that the overall heat transfer coefficients gradually increase with the number of repeated usage, $u$. Furthermore, the ratio of increase of the overall heat transfer coefficient of the ladle wall is the same as that of the ladle bottom. In addition, it is assumed that the temperature difference between the top and the bottom of ladle increases with the increase of $u$. The relations are expressed by

$$
\begin{aligned}
& U_{\mathrm{w}}(u)=\beta U_{\mathrm{b}}(u) \\
& U_{\mathrm{b}}(u)=U_{\mathrm{b} 0}+\gamma \sqrt{u} \\
& \alpha(u)=\alpha_{0}+\eta \sqrt{u}
\end{aligned}
$$

where $\beta, U_{\mathrm{b} 0}, \gamma, \alpha_{0}$ and $\eta$ are constants. 


\subsection{First-Principle Model for Casting Period}

\subsubsection{Molten Steel in Ladle}

It is assumed that the volumetric flow $Q$ from the ladle to the tundish is constant. Let $T_{\mathrm{md}}(z, t)$ be the temperature profile in the ladle during the discharging period, and the starting moment of discharging regarded as $t=0$. Then, the enthalpy balance of the molten steel in the ladle during the withdrawal period is given by the following equations.

$$
\begin{aligned}
& \rho_{\mathrm{m}} c_{\mathrm{m}} \pi R_{i}^{2} H_{\mathrm{m}} \frac{d \bar{T}_{\mathrm{m}}(t)}{d t}=-2 \pi R_{i} \int_{0}^{H_{\mathrm{m}}} U_{\mathrm{w}}\left(T_{\mathrm{md}}(z, t)-T_{\mathrm{am}}\right) d z \\
& -\pi R_{i}^{2} U_{\mathrm{b}}\left(T_{\mathrm{md}}(0, t)-T_{\mathrm{am}}\right)-\pi R_{i}^{2} h_{1}\left(T_{\mathrm{md}}\left(H_{\mathrm{m}} t\right)-T_{\mathrm{sl}}(t)\right) \\
& -\rho_{\mathrm{m}} c_{\mathrm{m}} Q\left(T_{\mathrm{md}}(0, t)-\bar{T}_{\mathrm{m}}(t)\right) \\
& H_{\mathrm{m}}(t)=H_{\mathrm{m}}(0)-\frac{Q}{\pi R^{2}} t \\
& T_{\mathrm{md}}(z, 0)=T_{\mathrm{m}}\left(z, t_{f}\right)
\end{aligned}
$$

where $t_{\mathrm{f}}$ is the ending moment of the transportation period. The last term of the right hand side of Eq. (23) shows the effect of withdrawal. $H_{\mathrm{m}}$ is gradually decreased by the withdrawal of the molten steel. It is assumed that the shape of the temperature profile in the ladle is not changed during the discharging period, i.e., the temperature profile during the discharging period is assumed to be given by the following equation:

$T_{\mathrm{md}}(z, t)=\bar{T}_{\mathrm{m}}(t)+T_{\mathrm{md}}\left(z+\frac{Q}{\pi R^{2}} t, 0\right)-\frac{1}{H_{\mathrm{m}}(t)} \int_{0}^{H_{\mathrm{m}}(t)} T_{\mathrm{md}}\left(z+\frac{R}{\pi R^{2}} t, 0\right) d z(26)$

The last term of Eq. (26) is added so that the average temperature of $T_{\mathrm{m}}$ becomes $\bar{T}_{\mathrm{m}}$. An example of temperature profiles at the starting moment and the middle of the discharging period is shown in Fig. 4. For the enthalpy balance of the slag layer, Eq. (19) is used. 


\subsubsection{Molten Steel in Tundish}

It is assumed that the inflow to the tundish is equal to the outflow from the tundish and also the depth of the molten steel in the tundish is constant. The CFD simulations have indicated that temperature in the tundish is distributed in the flow direction [15]. Thus, the tundish is modeled as a compartment model consisting of $N_{\mathrm{t}}$ isothermal baths connected in series as shown in Fig. 5. The heat balance of the $k$ th bath is

$$
\begin{gathered}
\rho_{\mathrm{m}} c_{\mathrm{m}} W H \frac{L}{N_{\mathrm{t}}} \frac{d T_{\mathrm{t}}^{(k)}(t)}{d t}=\rho_{\mathrm{m}} c_{\mathrm{m}} Q T_{\mathrm{t}}^{(k-1)}(t)-\rho_{\mathrm{m}} c_{\mathrm{m}} Q T_{\mathrm{t}}^{(k)}(t)-S_{\mathrm{t}} U_{\mathrm{t}}\left(T_{\mathrm{t}}^{(k)}(t)\right. \\
\left.-T_{\mathrm{am}}\right)-W \frac{L}{N_{\mathrm{t}}} \varepsilon_{\mathrm{t}} \sigma\left(T_{\mathrm{t}}^{(k)}(t)^{4}-T_{\mathrm{am}}^{4}\right)-W \frac{L}{N_{\mathrm{t}}} h_{3}\left(T_{\mathrm{t}}^{(k)}(t)-T_{\mathrm{a} 2}\right) \\
S_{t}= \begin{cases}W \frac{L}{N_{\mathrm{t}}}+2 H \frac{L}{N_{\mathrm{t}}}+W H & \left(k=1, N_{\mathrm{t}}\right) \\
W \frac{L}{N_{\mathrm{t}}}+2 H \frac{L}{N_{\mathrm{t}}} & \left(k=2,3, \cdots, N_{\mathrm{t}}-1\right)\end{cases}
\end{gathered}
$$

where $W, H$, and $L$ denote the width, the height, and the length of molten steel in the tundish, respectively. $T_{\mathrm{t}}^{(k)}$ is the tundish temperature in the $k$ th bath. $S_{\mathrm{t}}$ denotes the contact area between the molten steel and the tundish, $U_{\mathrm{t}}$ the overall heat transfer coefficient of the tundish, $\varepsilon_{\mathrm{t}}$ the emissivity of the molten steel, $T_{\mathrm{a} 2}$ the air temperature in the tundish, and $h_{3}$ the heat transfer coefficient between the molten steel and the air. $T_{\mathrm{t}}^{(0)}(t)$ is equal to $T_{\mathrm{in}}(t)$ because the molten steel poured from the ladle flows into the first bath. The left side of Eq. (27) represents the time change of the molten steel enthalpy. The first to fifth terms of the right side represent the inflow enthalpy, the outflow enthalpy, the heat conduction from the molten steel to the tundish wall, the radiation from the molten steel to the tundish wall and the heat conduction from the molten steel to the air in the tundish, respectively. The tundish wall temperature is assumed to be equal to the air temperature $T_{\text {am }}$, which is assumed to be constant. 


\subsection{Parameter Estimation}

The physical model contains 13 parameters to be estimated: $\alpha_{0}, \gamma, \eta, h_{1}$, $h_{2}, h_{3}, U_{\mathrm{b} 0}, U_{\mathrm{t}}, T_{\mathrm{a} 1}, T_{\mathrm{a} 2}, \varepsilon_{\mathrm{sl}}, \varepsilon_{\mathrm{t}}$ and $\beta$. The first ten parameters were identified through the least squares algorithm using real process data and $\varepsilon_{\mathrm{sl}}, \varepsilon_{\mathrm{t}}$ and $\beta$ were given in advance from engineers' experience. The dimensions of the ladle and the tundish and the physical properties of steel were also given in advance. $N_{\mathrm{t}}$ was set equal to three based on the CFD study conducted by Odenthal et al. [15]. The input variables of the physical model are the number of ladle usage, the weight of the molten steel in the ladle, the initial temperature of steel in the ladle, the transportation time, the casting flow rate, and the initial temperature of each bath composing the tundish. The output variable is the temperature of the last bath, TD temp. A total of 1270 samples were used for parameter estimation.

\section{Prediction and Control of Molten Steel Temperature in Tundish}

In this section, three types of gray-box models, i.e., the parallel, the serial, and the combined gray-box models, are constructed to predict and control TD temp.

\subsection{Parallel Gray-box Model}

In the parallel gray-box model, a statistical model to compensate the prediction error of the first-principle model was developed by using 53 process variables, measured at the processes from the converter to the tundish, including the variables used in the first-principle model.

To build the statistical model, random forests (RF) [16] was used. RF is an ensemble classifier that consists of many decision trees. RF combines Breiman's bagging idea and the random selection of split features [17, 18]. Given a training set $D$ of size $N$, bagging generates $J$ new training sets $D_{j}^{*}(j=1,2, \cdots, J)$, whose size is $N$, by random sampling from $D$ with replacement. The set $D_{j}^{*}$ is expected to have about two-thirds of the unique 
datasets in $D$ and the rest is duplicated. In addition, at each node of the tree, feature variables, i.e., split features, are randomly selected and splitting is performed using these features one by one to find the best split. The sampled training datasets are called bootstrapped samples, while the fraction of original data that is not bootstrapped is termed out-of-bag (OOB) data. $\mathrm{RF}$ creates multiple trees; each tree is trained by using the bootstrapped samples. RF for regression is formed by growing trees on $(\boldsymbol{x}, y) \in D_{j}^{*}$ such that the predictions $\hat{f}(\boldsymbol{x})$ are numerical values as opposed to class labels in classification. OOB data is used for error calculation of the respective trees. The output of an RF model is the average of predicted values of all trees. In the RF model used in the parallel gray-box model, the number of trees and split features was set at the optimized values of 1000 and 13, respectively.

\subsection{Serial Gray-box Model}

The prediction performance of the first-principle model might be improved by taking account of the dependence of the parameters on the process conditions. That is, a serial gray-box model might be effective at reducing the prediction errors. However, it is not clear which parameter should be updated according to changes in process conditions. Thus, each of all the parameters was regarded as a candidate variable to be expressed as a function of measured process variables, and the most influential parameter was selected. For this purpose, mean absolute error (MAE) was calculated for each parameter.

$\mathrm{MAE}_{i}=\frac{1}{N_{\mathrm{d}}} \sum_{n=1}^{N_{\mathrm{d}}}\left|y_{n}-f_{\mathrm{fp}}\left(\boldsymbol{x}_{\mathrm{fp}, n}, \tilde{\boldsymbol{\theta}}_{i}^{c}, \tilde{\theta}_{i, n}\right)\right|$

where $\tilde{\theta}_{i, n}$ is the optimal value of $\theta_{i, n}$ which is derived by Eqs. (8) to (9). Since $U_{\mathrm{b} 0}$ is supposed to be a constant value, Eq. (21) was discarded and $U_{\mathrm{b}}$ was regarded as a parameter instead of $U_{\mathrm{b} 0}$ and $\gamma$. Thus, the number of parameters in the first-principle model, $N_{\mathrm{p}}$, is eight in this case. Table 1 
shows MAEs after optimizing the parameters of the first-principle model one by one. It is clear that the error was drastically reduced when the overall heat transfer coefficients of the ladle bottom $U_{\mathrm{b}}$ was optimized. This result is consistent with the knowledge of process engineers and plant operators; the heat loss from the ladle is a key factor to model the process, and it is strongly affected by the ladle degradation. Thus, $U_{\mathrm{b}}$ was selected as the variable to be updated as a function of 53 process variables. The optimal value of $U_{\mathrm{b}}$ was calculated for each modeling sample, and a statistical model was developed by RF to estimate $U_{\mathrm{b}}$. In this RF model, the number of trees and the split features was set at the same values as that of the RF model in the parallel gray-box model. Fig. 6 shows the relationship between the optimized values of $U_{\mathrm{b}}$ and the training errors of the first-principle model. The positive error shows that the heat loss calculated by the first-principle model is larger than the actual heat loss in the plant while the negative error shows that the heat loss calculated by the first-principle model is smaller than the actual heat loss in the plant. To precisely model the heat loss, $U_{\mathrm{b}}$ should be decreased for positive error and increased for negative error.

\subsection{Combined Gray-box Model}

By combining an outer statistical model with the serial gray-box model, a combined gray-box model was developed. The outer statistical model was built by applying RF to the data of the prediction error of the serial gray-box model and the measurements of 53 process variables. In addition, a statistical model was developed by using only RF to evaluate the performance of the statistical model, whose inputs were 53 process variables.

The prediction performance of the first-principle model, the statistical (RF) model, the parallel gray-box model, the serial gray-box model, and the combined gray-box model, was compared by applying them to real operation data of a steel making plant. The total number of samples was 1588; 1270 samples (80\%) were used for modeling and the remaining 318 samples $(20 \%)$ were used for validation. The prediction results are shown in 
Fig. 7. The prediction performance was evaluated on the basis of the rootmean-square error (RMSE) and the correlation coefficient (r) between scaled values of the reference TD temp and the predicted TD temp. RMSE of the first-principle model, the RF model, the parallel gray-box model, the serial gray-box model, and the combined gray-box model is $2.73,2.08,1.83,1.81$ and 1.74, respectively. The performance of the proposed combined gray-box model is superior to the other models. The RMSE of the combined gray-box model is $36 \%, 16 \%, 5 \%$ and $4 \%$ smaller than that of the first-principle model, the RF model, the parallel gray-box model, and the serial gray-box model, respectively. Although the serial (inner) gray-box model performs better than the first-principle model and the RF model significantly, there remain large prediction errors. Such prediction errors cannot be compensated by updating the parameters of the first-principle model, because the structure of the model imposes limitations on the prediction performance. Additionally, the errors caused by process uncertainties in temperature measurements, composition and weight of added alloys, and the extent of oxidation reactions for removal of impurities cannot be compensated by updating the parameters. On the other hand, the combined gray-box model uses the additional statistical model to compensate such prediction errors and therefore outperforms all the models including the serial gray-box model.

\subsection{Control of TD temp}

Since the combined gray-box model was able to predict TD temp accurately, the next step was to adjust $\mathrm{RH}$ temp in order to realize precise control of TD temp. Thus, the developed prediction model was used as the control model for feed-forward control, in which the manipulated variable, RH temp, was optimized with the bisection method in order to achieve the target TD temp. Three different values of RH temp, i.e., RH-max, RH-mid and RHmin, are determined. Initially, TD temp is predicted through the combined gray-box model using RH-mid. Then the value of $\mathrm{RH}$ temp is iteratively updated using the bisections method and new TD temp is predicted until 
it becomes equal to the target TD temp. Fig. 8 shows the results of $\mathrm{RH}$ temp derivation by using the first-principle model, the RF model, the parallel gray-box model, the serial gray-box model, and the combined gray-box model. The performance was evaluated on the basis of the root-mean-square error (RMSE) and the correlation coefficient $(r)$ between scaled values of the reference $\mathrm{RH}$ temp and the derived $\mathrm{RH}$ temp. RMSE of the first-principle model, the RF model, the parallel gray-box model, the serial gray-box model, and the combined gray-box model is $2.90,1.65,1.95,1.80$ and 1.64, respectively. The performance of the proposed combined gray-box model is superior to the other models. The combined gray-box model achieved the highest prediction accuracy and its RMSE is $43 \%, 1 \%, 16 \%$ and $10 \%$ smaller than those of the first-principle model, the RF model, the parallel gray-box model, and the serial gray-box model, respectively.

\section{Conclusions}

In the steel making plant, the molten steel temperature in tundish of continuous casting processes (TD temp) is one of the key factors to realize stable operation, and it is controlled by manipulating the molten steel temperature in the secondary refining process ( $\mathrm{RH}$ temp).

In this research, new gray-box models were developed to predict and control TD temp with high accuracy, because the performance of the adopted first-principle model (physical model) was not sufficient. In the serial graybox model, the overall heat transfer coefficient of the ladle bottom was optimized for each past batch separately and updated as a function of 53 process variables with random forests $(\mathrm{RF})$. The prediction errors of the firstprinciple model and those of the serial gray-box model were compensated by using outer statistical models; this approach derived the parallel gray-box model and the combined gray-box model. All statistical models were built by using RF. The developed models were used not only for the prediction of TD temp but also for its control. For this purpose, the proposed gray-box 
models were inversely used to derive RH temp from the desired TD temp.

The results of TD temp prediction and RH temp derivation have demonstrated the advantage of the combined gray-box model over the first-principle model, the statistical model, and the conventional gray-box models. For example, the combined gray-box model realized $36 \%$ and $16 \%$ smaller RMSEs of TD temp prediction than the first-principle model and the RF model, respectively. Moreover, the combined gray-box model achieved 16\% and $10 \%$ smaller RMSEs of RH temp derivation than the parallel gray-box model and the serial gray-box model, respectively. The performance of the combined gray-box model satisfies the requirement for its industrial application; thus it is expected that the developed system can be used as a part of an operation support system of the target steel making plant.

\section{Acknowledgments}

This study is partially supported by the grant from ISIJ as an activity of research group, High Precision Process Control via Large Scale Database and Simulation Models, and also by Japan Society for the Promotion of Science (JSPS), Grant-in-Aid for Scientific Research (C) 24560940.

\section{References}

[1] M. Kano, Y. Nakagawa, Data-based process monitoring, process control, and quality improvement: Recent developments and applications in steel industry, Computers \& Chemical Engineering 32 (1-2) (2008) 12-24.

[2] A. Zoryk, P. M. Reid, On-line liquid steel temperature control, Iron and Steelmaker 20 (1993) 21-27.

[3] A. G. Belkovskii, Y. L. Kats, Mathematical model of the cooling of steel in a small ladle, Metallurgist 53 (2009) 261-273. 
[4] P. R. Austin, J. M. Camplin, J. Herbertson, I. J. Taggart, Mathematical modeling of thermal stratification and drainage of steel ladles, ISIJ International 32 (2) (1992) 196-202.

[5] J. L. Xia, T. Ahokainen, Transient flow and heat transfer in a steelmaking ladle during the holding period, Metallurgical and Materials Transactions B 32 (2001) 733-741.

[6] J. R. S. Zabadal, M. T. M. B. Vilhena, S. Q. B. Leit, Heat transfer process simulation by finite differences for online control of ladle furnaces, Ironmaking and Steelmaking 31 (2004) 227-232.

[7] T. Jormalainen, S. Louhenkilpi, A model for predicting the melt temperature in the ladle and in the tundish as a function of operating parameters during continuous casting, Steel Research International 77 (2006) 472-484.

[8] S. Sonoda, N. Murata, H. Hino, H. Kitada, M. Kano, A statistical model for predicting the liquid steel temperature in ladle and tundish by bootstrap filter, ISIJ International 52 (2012) 1086-1091.

[9] T. A. Johansen, B. A. Foss, Representing and learning unmodelled dynamics with neural network memories, American Control Conference, (1992) 3037-3043

[10] D. C. Psichogios, L. H. Ungar, A hybrid neural network first principles approach to process modeling, AIChE Journal, 38 (10) (1992) 1499-1511.

[11] N. Gupta, S. Chandra, Temperature prediction model for controlling casting superheat temperature, ISIJ International 44 (2004) 1517-1526.

[12] T. Okura, I. Ahmad, M. Kano, S. Hasebe, H. Kitada, N. Murata, Highperformance prediction of molten steel temperature in tundish through gray-box model, ISIJ International 53 (1) (2013) 76-80. 
[13] T. P. Fredman, Heat transfer in steelmaking ladle refractories and steel temperature, Scandinavian Journal of Metallurgy 29 (2000) 232-258.

[14] A. Tripathi, J. K. Saha, J. B. Singh, S. K. Ajmani, Numerical simulation of heat transfer phenomenon in steel making ladle, ISIJ International 52 (9) (2012) 1591-1600.

[15] H. J. Odenthal, M. Javurek, M. Kirschen, N. Vogl, CFD benchmark for a single strand tundish, Steel Research International 81 (7) (2010) 529-541.

[16] L. Breiman, Random forests, Machine Learning 45 (2001) 5-32.

[17] T. K. Ho, Random decision forest, Proceedings of 3rd International Conference on Document Analysis and Recognition, Canada, 1 (1995) 278282.

[18] T. K. Ho, The random subspace method for constructing decision forests, IEEE Transactions on Pattern Analysis and Machine Intelligence 20 (8) (1998) 832-844. 


\section{Figure captions}

Figure 1 Schematic diagram of the steel making process.

Figure 2 Generalized framework of gray-box modeling.

Figure 3 Model of molten steel temperature in ladle during the transportation period from the secondary refining process to the continuous casting process. Figure 4 Model of molten steel temperature in ladle during the casting period. Figure 5 Compartment model of the tundish.

Figure 6 Training errors of the first-principle model and the corresponding optimized values of the overall heat transfer coefficient $U_{\mathrm{b}}$.

Figure 7 Prediction of TD temp through the first-principle model, the RF model, the parallel gray-box model, the serial gray-box model, the combined gray-box model, and predicted and reference TD temp.

Figure 8 Derivation of $\mathrm{RH}$ temp through the first-principle model, the RF model, the parallel gray-box model, the serial gray-box model, the combined gray-box model, and derived and reference RH temp. 


\section{Table captions}

Table 1 Mean absolute error (MAE) after optimizing each parameter in the first-principle model. 


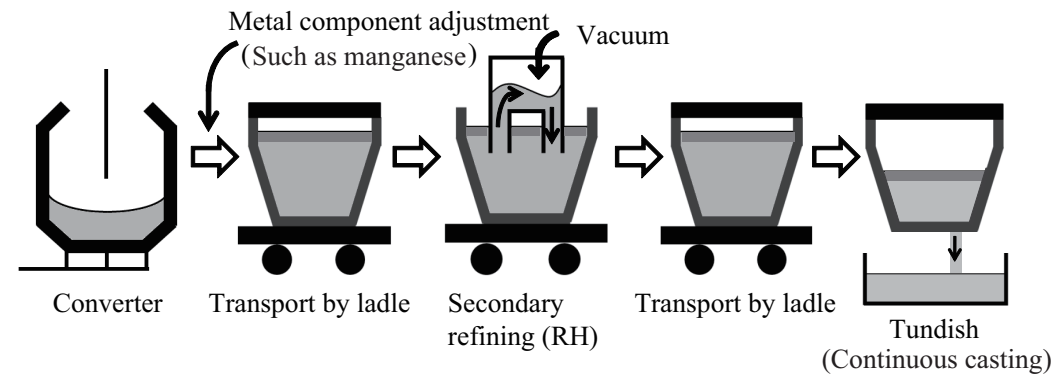

Figure 1: Schematic diagram of the steel making process 


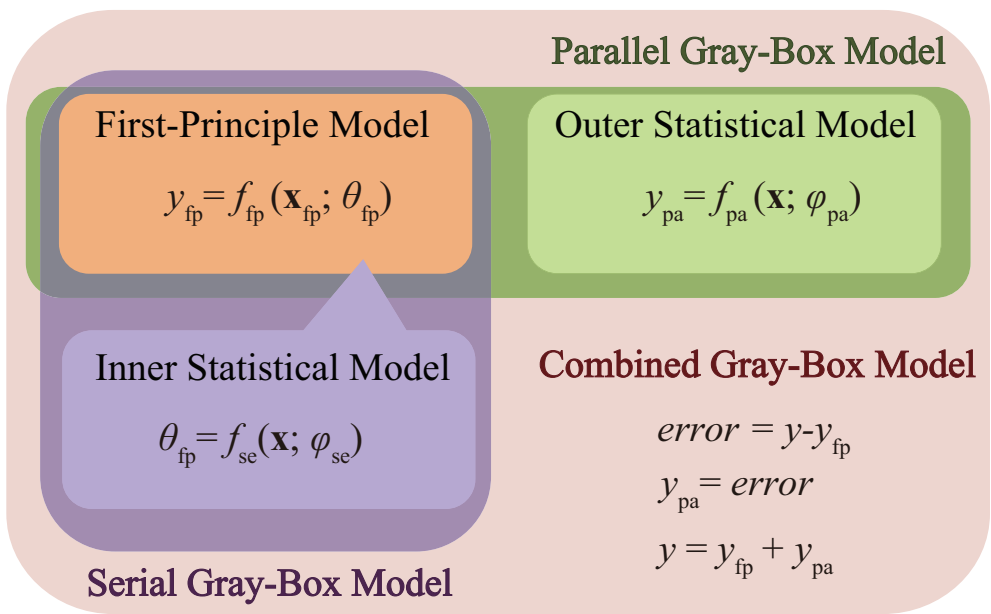

Figure 2: Generalized framework of gray-box modeling 


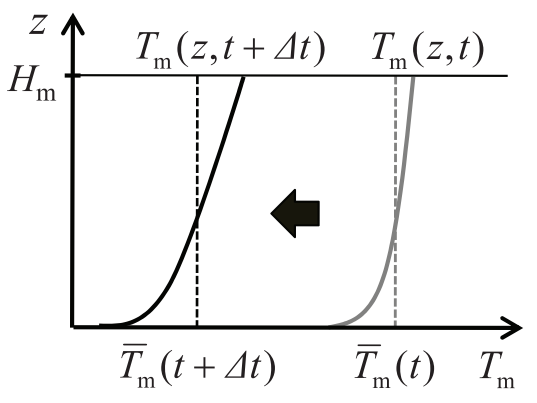

Figure 3: Model of molten steel temperature in ladle during the transportation period from the secondary refining process to the continuous casting process 

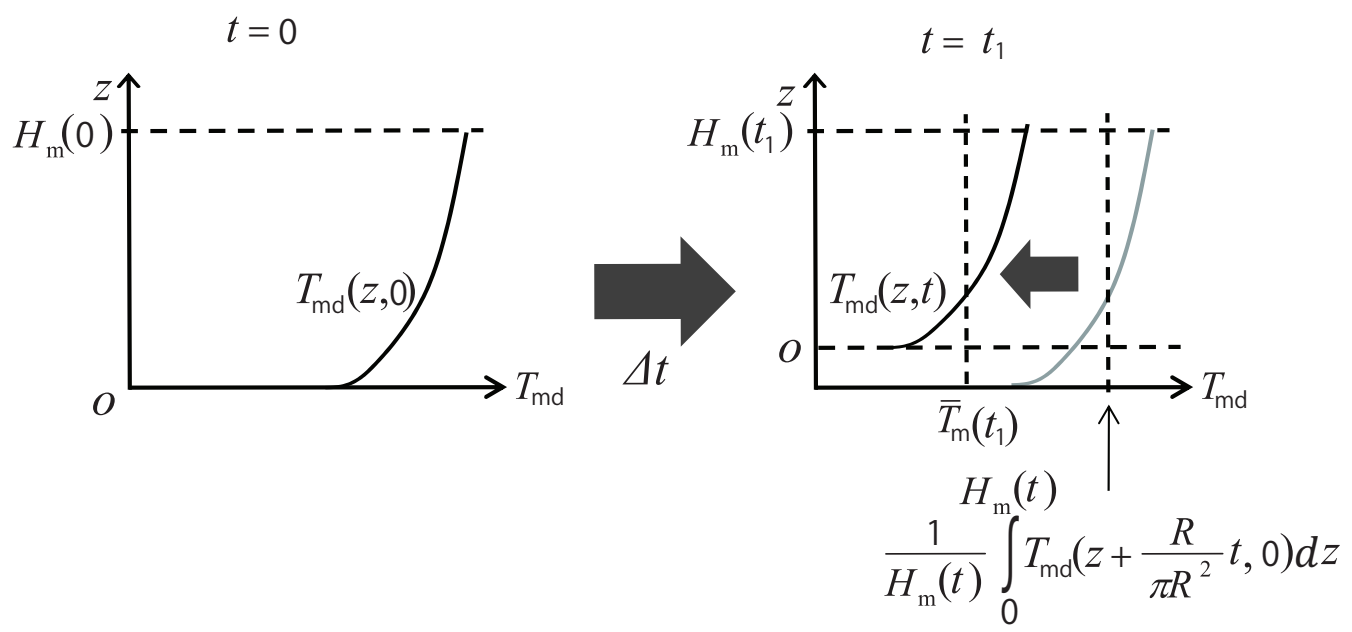

Figure 4: Model of molten steel temperature in ladle during the casting period 



Figure 5: Compartment model of the tundish 


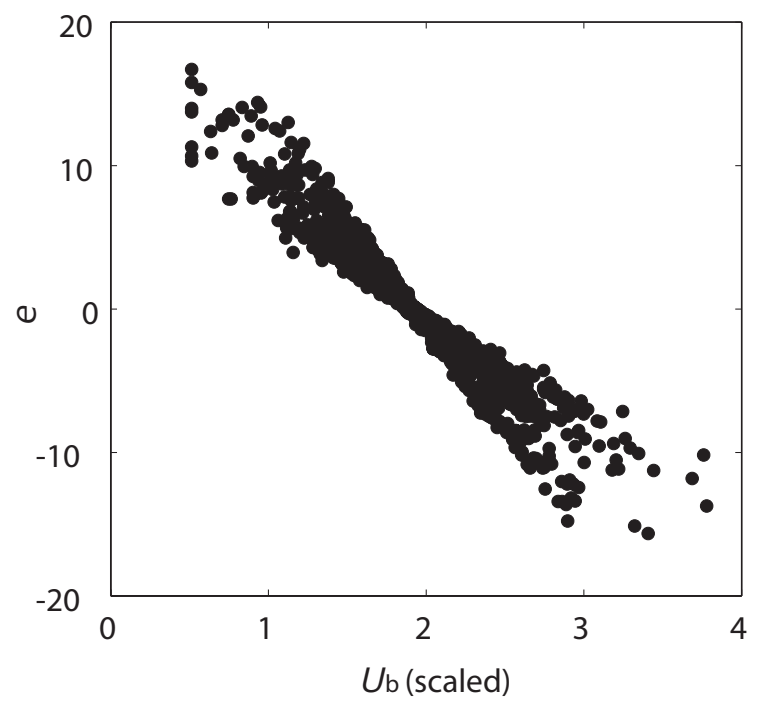

Figure 6: Training errors of the first-principle model and the corresponding optimized values of the overall heat transfer coefficient $U_{\mathrm{b}}$ 
Table 1: Mean absolute error (MAE) after optimizing each parameter in the first-principle model

\begin{tabular}{cc}
\hline \hline Parameter & MAE \\
\hline$\alpha_{0}$ & 1.28 \\
$\gamma$ & 1.50 \\
$\eta$ & 1.94 \\
$h_{1}$ & 2.29 \\
$h_{2}$ & 3.84 \\
$h_{3}$ & 3.71 \\
$U_{\mathrm{b}}$ & $3.30 \times 10^{-5}$ \\
$U_{\mathrm{t}}$ & 1.51 \\
$T_{\mathrm{a} 1}$ & 1.85 \\
$T_{\mathrm{a} 2}$ & 0.03 \\
\hline \hline
\end{tabular}



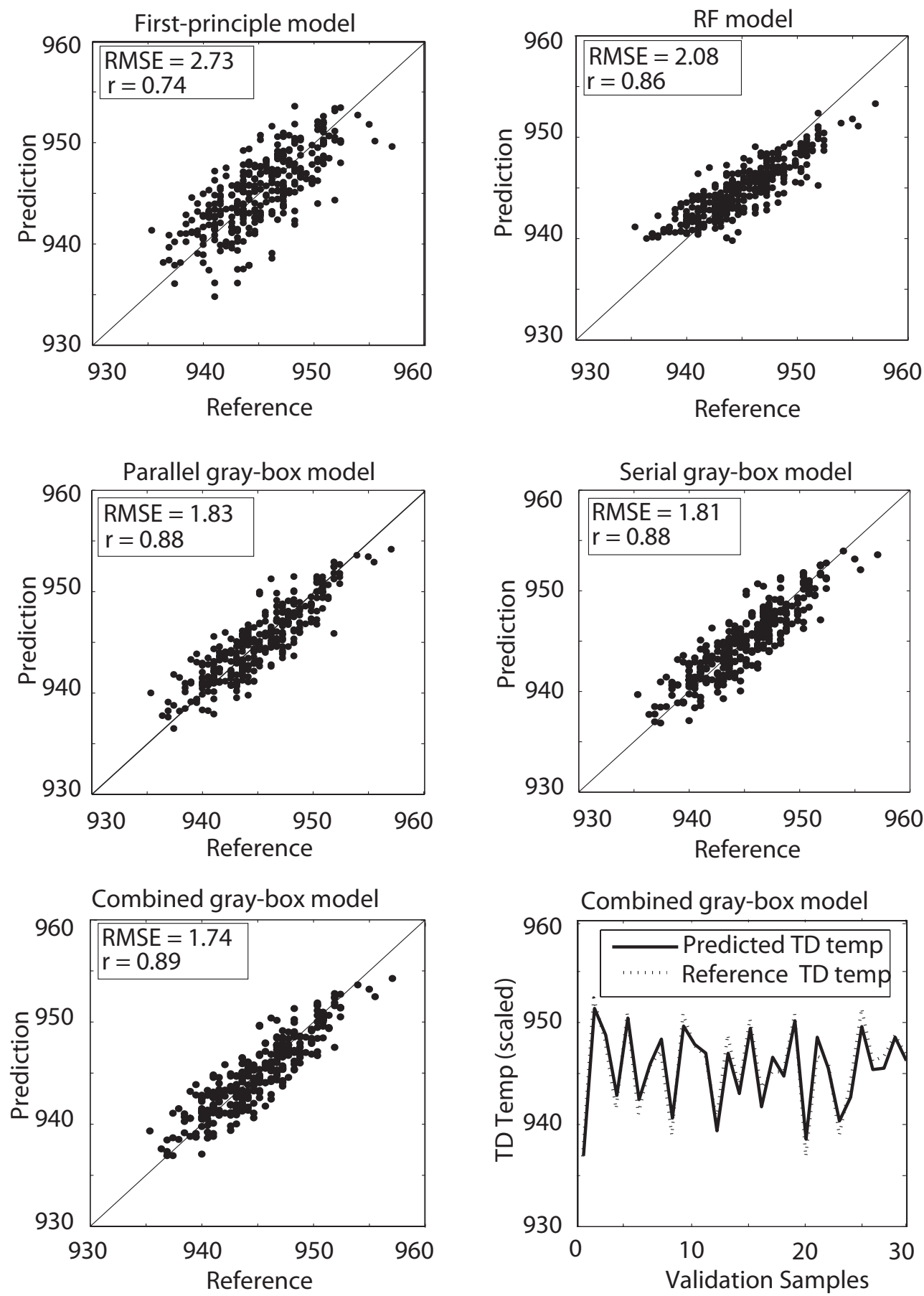

Figure 7: Prediction of TD temp through the first-principle model, the RF model, the parallel gray-box model, the serial gray-box model, the combined gray-box model, and predicted and reference TD temp 

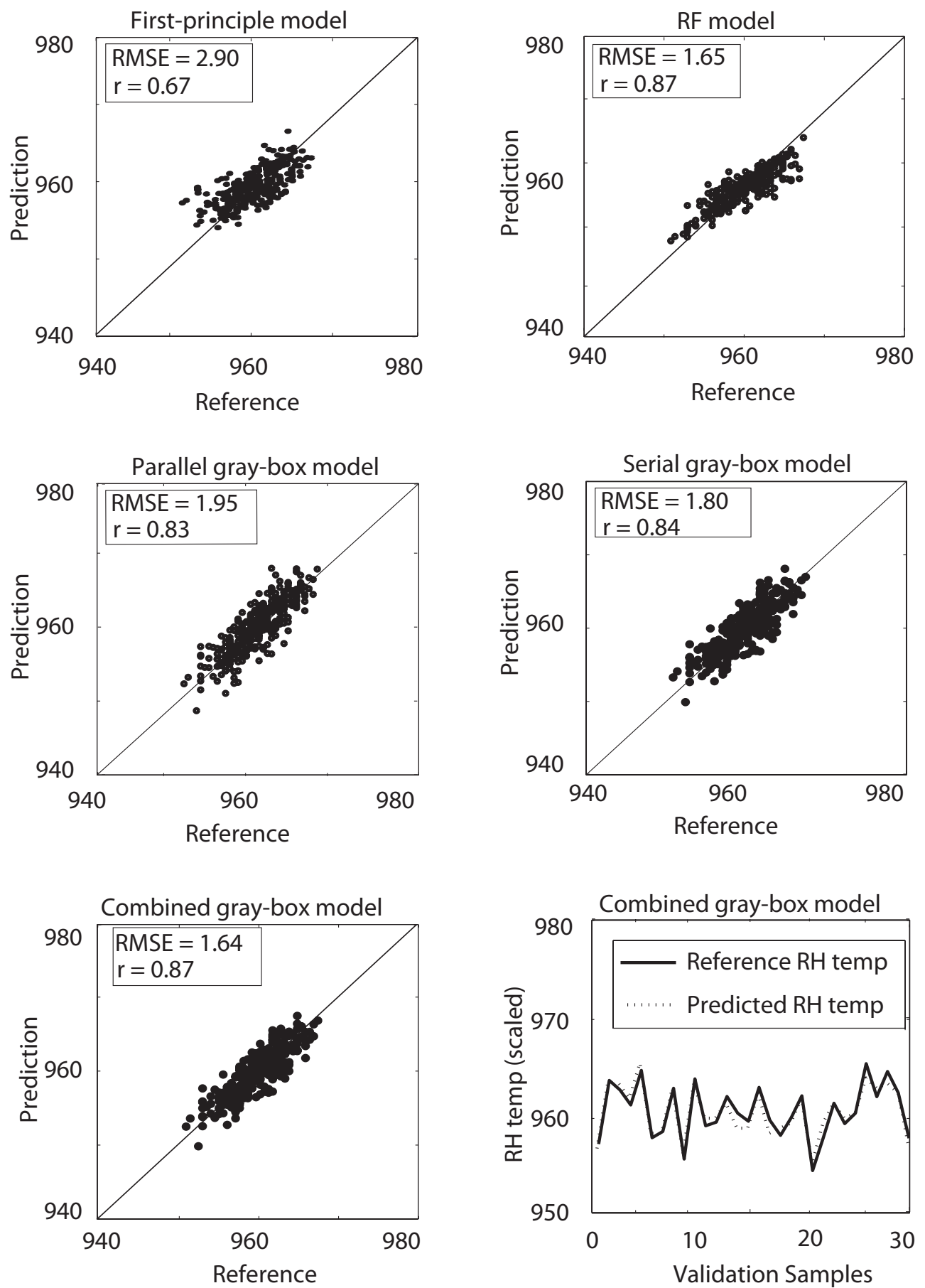

Figure 8: Derivation of RH temp through the first-principle model, the RF model, the parallel gray-box model, the serial gray-box model, the combined gray-box model, and derived and reference RH temp 